\title{
Application of the general matching law on the study of multi- coated radar absorbing materials
}

\author{
Haifeng Zhang, Jiubo Cui, Weiwei Pei*, and Dongfang Wang* \\ College of Science, Jiamusi University, 154007 Jiamusi, China
}

\begin{abstract}
In this paper, the Generalized Matching Law of electromagnetic parameters was given for in the application of radar absorbing materials, and applied in the research of absorbing properties of multi-coated absorbing materials. We gave the power reflection formula, defined the constant of general matching law $\mathrm{M}$, and discussed the relationship of general matching constant $M$ with power reflectivity $R_{P}$, thickness $d$, relative permittivity $\varepsilon_{\mathrm{r}}$, relative permeability $\mu_{\mathrm{r}}$. We found that the enhancement of RP was not obvious at the $\varepsilon^{\prime \prime}$ was optimized, but the $\mu^{\prime \prime}{ }_{\mathrm{r}}$ was primary. In short, this is consistent with panorama analysis method.
\end{abstract}

\section{Introduction}

The radar signature is given by the radar cross section (RCS) that can be viewed as a comparison of the strength of the reflected signal from a target to the reflected signal ${ }^{[1]}$.

Radar absorbing materials (RAM) can be divided into coating material, structure composites, patch material and other type of absorbing materials ${ }^{[2]}$. Rubber radar absorbing materials is mainly made of rubber matrix and electromagnetic wave absorbents. The absorbents provide the necessary electromagnetic performances of the RAM ${ }^{[3-6]}$.

Carbonyl iron can be used as a good microwave absorbent in 1-18 $\mathrm{GHz}$ and even a higher frequency range, and it has been used in the field of electromagnetic shielding and absorbing materials widely $^{[4-5]}$.

The camouflage of aerial, marine and ground targets are of great interest today when the radar technology has reached a very high level. And, in other types of target camouflage, the use of RAM is more and more feasibly at least for ground targets ${ }^{[6]}$.

A RAM must assure an attenuation of electromagnetic waves and a good match at the air/material interface and these involve that the material must satisfy two contradictory conditions ${ }^{[7-9]}$.

The object is to match the impedance of the air at the front surface, and slowly taper this impedance to a very low value, approaching a short circuit, at the back surface $^{[8]}$. Most military applications require thin absorbent coating. A thin absorber can be theoretically designed to give any reflectivity but it is very difficult to manufacture. Multilayer RAMs have the best performances but they are complicated to design ${ }^{[9-10]}$.

\section{The discussion on the GML}

In the study of thin coating of RAM, the proposition of the general matching law (GML) and the panorama analysis method(PAM) were instructive on the development of high-level coating materials, the effective selection of materials and the optimized design of materials.

Studies had shown that, to make the most energy flow into the medium at the limited medium thickness, the electromagnetic parameters of the medium should satisfy the GML, such as $\varepsilon_{\mathrm{r}}^{\prime} \mu^{\prime \prime}{ }_{\mathrm{r}}-\varepsilon_{\mathrm{r}}{ }_{\mathrm{r}} \mu_{\mathrm{r}}^{\prime}=0$.

So, we set $\varepsilon_{\mathrm{r}}^{\prime \prime} / \varepsilon_{\mathrm{r}}^{\prime}=\mu_{\mathrm{r}}^{\prime \prime} / \mu_{\mathrm{r}}^{\prime}=\mathrm{M}$, and defined the $\mathrm{M}$ as the generalized matching constant ${ }^{[11-13]}$.

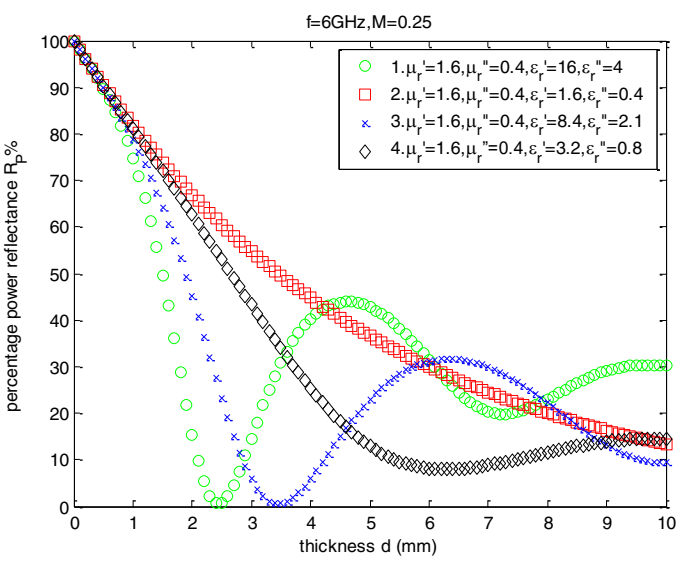

Fig.1. The $\mathrm{R}_{\mathrm{P}} \%$-d curves at the given $\varepsilon_{\mathrm{r}}^{\prime}, \varepsilon_{\mathrm{r}}^{\prime \prime}, \mu_{\mathrm{r}}^{\prime}$ and $\mu_{\mathrm{r}}^{\prime \prime}$ values

In figure 1, the relationship curve between the percentage power reflectance $\mathrm{R}_{\mathrm{P}} \%$ and the thickness was given, at $\mathrm{M}=0.25, \mathrm{f}=6 \mathrm{GHz}^{[2]}$. Here, we plotted the curves of the $R_{P} \%$ and $d$ at different $M$ values in the figure 2. Through the analysis of the curves, it was

\footnotetext{
* Corresponding author: peiweiwei50082@126.com;

jmsdxwdf2012@126.com
} 
obvious that if $\mathrm{M}$ took the different values, the decline rates of curves were different significantly.

At the meanwhile, the figure 2 showed that the greater of $\mathrm{M}$ would lead to the rapidly decreasing rate of the curve, and some of the curves would be oscillated. So we believed that there should be a corresponding reflection formula to match with the GML ${ }^{[14-16]}$.

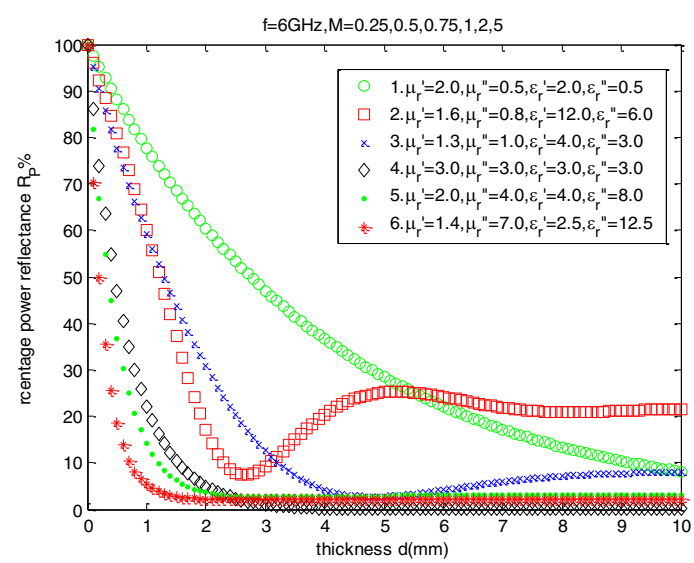

Fig.2. The Re\%-d curves at the given $\varepsilon_{\mathrm{r}}^{\prime}, \varepsilon_{\mathrm{r}}^{\prime \prime}, \mu_{\mathrm{r}}^{\prime}, \mu_{\mathrm{r}}^{\prime \prime}$ and $\mathrm{M}$ values

\section{Derivation of the reflectivity formula}

In order to a facilitate discussion, we first derived a single layer medium situation with the metal base. As we known that when the electromagnetic waves interacted with the media, the reflection coefficient was defined as $^{[17]}$

$$
R_{1}=\left|\frac{Z_{1, \text { in }}-Z_{0}}{Z_{1, \text { in }}+Z_{0}}\right|
$$

Where, $Z_{1, \text { in }}$ was the input impedance of the dielectric layer. The relationship between the electromagnetic parameters of the material under the GML is $\varepsilon_{r}^{\prime} \mu^{\prime \prime}{ }_{r}^{-}$ $\varepsilon{ }_{\mathrm{r}}^{\prime \prime} \mu_{\mathrm{r}}^{\prime}=0$, so we set

$$
\varepsilon_{r 1}^{\prime \prime} / \varepsilon_{r 1}^{\prime}=\mu_{r 1}^{\prime \prime} / \mu_{r 1}^{\prime}=M_{1}
$$

As we known that when the electromagnetic waves propagate in lossy media, the wave vector is complex

$$
k_{1}=k_{1}^{\prime}-\mathrm{j} k_{1}^{\prime \prime}
$$

Where, the $k_{1}^{\prime}$ and $k_{1}^{\prime \prime}$ are respectively,

$$
\begin{gathered}
k_{1}^{\prime}=\frac{2 \pi f}{c} \sqrt{\varepsilon_{r 1}^{\prime} \mu_{r 1}^{\prime}} \sqrt{\frac{1}{2}[1-A+\sqrt{B}]} \\
k_{1}^{\prime \prime}=\frac{2 \pi f}{c} \sqrt{\varepsilon_{r 1}^{\prime} \mu_{r 1}^{\prime}} \sqrt{\frac{1}{2}[A-1+\sqrt{B}]} \\
A=\tan \delta_{e 1} \operatorname{tg} \delta_{m 1} \\
B=\left(1+\tan ^{2} \delta_{e 1}\right)\left(1+\tan ^{2} \delta_{m 1}\right)
\end{gathered}
$$

Where, the $\tan \delta_{\mathrm{e} 1}$ and $\tan \delta_{\mathrm{m} 1}$ are the tangent of electric loss angle and the tangent of magnetic loss angle respectively,

$$
\tan \delta_{e 1}=\varepsilon_{r 1}^{\prime \prime} / \varepsilon_{r 1}^{\prime}
$$

$$
\tan \delta_{m 1}=\mu_{r 1}^{\prime \prime} / \mu_{r 1}^{\prime}
$$

Therefore, taking equation (2), (8), (9) into (4), (5) would abtain

$$
\begin{gathered}
k_{1}^{\prime}=\frac{2 \pi f}{c} \sqrt{\varepsilon_{r 1}^{\prime} \mu_{r 1}^{\prime}} \\
k_{1}^{\prime \prime}=\frac{2 \pi f}{c} \sqrt{\varepsilon_{r 1}^{\prime} \mu_{r 1}^{\prime}} M_{1} \\
k_{1}=k_{1}^{\prime}-\mathrm{j} k_{1}^{\prime \prime}=\frac{2 \pi f}{c} \sqrt{\varepsilon_{r 1}^{\prime} \mu_{r 1}^{\prime}}\left(1-\mathrm{j} M_{1}\right)
\end{gathered}
$$

And since the input impedance of absorbing medium in the metal base would be expressed as

$$
Z_{1, \mathrm{in}}=\mathrm{j} Z_{1} \frac{\sin k_{1} d_{1}}{\cos k_{1} d_{1}}=Z_{1} \tanh \left(\mathrm{j} k_{1} d_{1}\right)
$$

the characteristic impedance of the media under the GML can be written as the following form

$$
Z_{1}=Z_{0} \sqrt{\frac{\mu_{r 1}^{\prime}}{\varepsilon_{r 1}^{\prime}}} \text { or } Z_{1}=Z_{0} \sqrt{\frac{\mu_{r 1}^{\prime \prime}}{\varepsilon_{r 1}^{\prime \prime}}}
$$

And the input impedance of lossy media should be plural, which is,

$$
Z_{1, \text { in }}=Z_{1, \text { in }}^{\prime}-\mathrm{j} Z_{1, \text { in }}^{\prime \prime}
$$

From the above derivation, we can see that

$$
\begin{gathered}
Z_{1, \text { in }}=Z_{1} \tanh \left(\mathrm{j} k_{1} d_{1}\right)=Z_{0} \sqrt{\mu_{r 1}^{\prime} / \varepsilon_{r 1}^{\prime}} \tanh C \\
C=\mathrm{j} \frac{2 \pi f d_{1}}{c}\left(1-\mathrm{j} M_{1}\right) \sqrt{\varepsilon_{r 1}^{\prime} \mu_{r 1}^{\prime}}
\end{gathered}
$$

So, the equation (16) into (1) will obtain the reflection coefficient formula

$$
R_{1}=\left|\frac{\sqrt{\mu_{r 1}^{\prime} / \varepsilon_{r 1}^{\prime}} \tanh C-1}{\sqrt{\mu_{r 1}^{\prime} / \varepsilon_{r 1}^{\prime}} \tanh C+1}\right|
$$

The corresponding power reflectivity $\mathrm{R}_{\mathrm{P} 1}(\mathrm{~dB})$ can be given by

$$
R_{p 1}=20 \lg \left(R_{1}\right)
$$

For the multilayer dielectric in the metal base, the derivation process of the GML shows that, according to the reference ${ }^{[1]}$, if we want to make the maximum energy flow penetration into each layer of the multi-media, each layer should satisfy the GML, which is ${ }^{[1]}$

$$
\varepsilon_{r i}^{\prime \prime} / \varepsilon_{r i}^{\prime}=\mu_{r i}^{\prime \prime} / \mu_{r i}^{\prime}=M_{i}
$$

Among them, $\mathrm{i}=1,2, \ldots, \mathrm{n}$. So the iterative formula of the nth layer medium is

$$
\begin{gathered}
Z_{n, \mathrm{in}}=\frac{Z_{(n-1), \mathrm{in}}+Z_{n} \tanh \left(\mathrm{j} k_{n} d_{n}\right)}{Z_{n}+Z_{(n-1), \mathrm{in}} \tanh \left(\mathrm{j} k_{n} d_{n}\right)} Z_{n} \\
k_{n}=k_{n}^{\prime}-\mathrm{j} k_{n}^{\prime \prime}=\frac{2 \pi f}{c} \sqrt{\varepsilon_{r n}^{\prime} \mu_{r n}^{\prime}}\left(1-\mathrm{j} M_{n}\right) \\
Z_{n}=Z_{0} \sqrt{\frac{\mu_{r n}^{\prime}}{\varepsilon_{r n}^{\prime}}}
\end{gathered}
$$

So, the reflection coefficient and reflectance formula of multi coating materials coating can be given 


$$
\begin{aligned}
& R_{n}=\left|\frac{Z_{n, \text { in }}-Z_{0}}{Z_{n, \text { in }}+Z_{0}}\right| \\
& R_{p n}=20 \lg \left(R_{n}\right)
\end{aligned}
$$

\section{Discussion and Conclusion}

According to the above derived formula of the reflectivity, we draw the curves among the $\mathrm{M}, \mathrm{R}_{\mathrm{P}}, \mathrm{R}_{\mathrm{P}} \%$, $\mathrm{d}, \varepsilon_{\mathrm{r}}$ and $\mu_{\mathrm{r}}$ in Figure 3-7. Here we discussed the various relations among them.

From figure 2, we can see that, with the thickness $d$ increases, at $\mathrm{M}>1$, the $\mathrm{R}_{\mathrm{P}} \%$ declines rapidly in the beginning, and soon tends to zero. At $\mathrm{M}<1$, it also declines rapidly, but with the emergence of oscillation the $d$ increases and tends to a certain value. And while the inpedance of the air and the media will be up to an exact matching, the oscillations disappear. The $M=1$ is a critical situation.

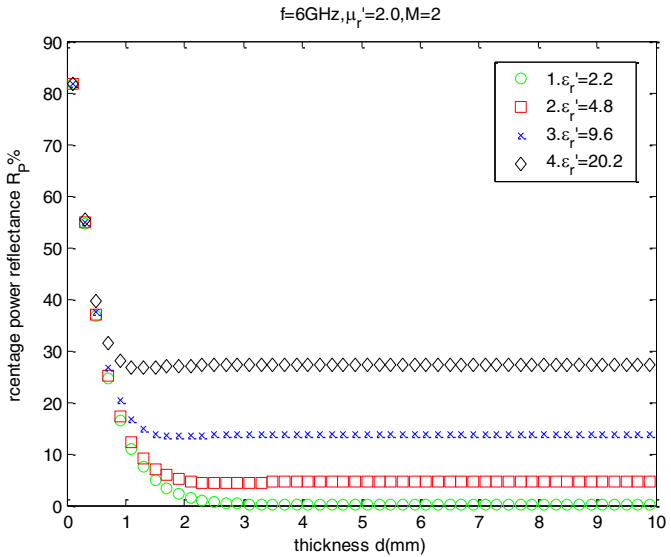

Fig.3. The $\mathrm{RP}_{\mathrm{P}} \%$-d curves at the given $\varepsilon_{\mathrm{r}}^{\prime}$ values

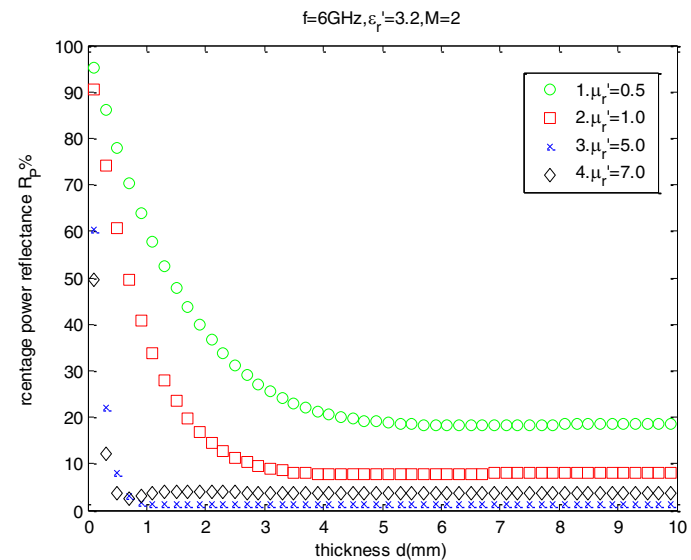

Fig.4. The Rp\%-d curves at the given $\mu_{\mathrm{r}}^{\prime}$ values

From figure 3 and 4, we can see that in a very small $d$ and the request of $1 \leq \mu_{\mathrm{r}}^{\prime} \leq 4$, a greater than 1 of $\mathrm{M}$ value should be taken if we want to make a greater decibel value. And the optimization of $\varepsilon^{\prime \prime}$ for the increase of $R_{P}$ is not obvious, while the optimization of $\mu_{r}{ }_{r}$ is main. This conclusion also proved the main point in the PAM.

In figure 5 , it is in the band of $1-14 \mathrm{GHz}$, and a thin material of $1.0 \mathrm{~mm}$. Obviously, in the high frequency range, taking the value of less than 1 of $\mathrm{M}$ will be able to reach the required reflectivity decibels. But, the $M$ value is demanded to be greater than 1 for matching the required reflectivity at the low frequency and the same thickness.

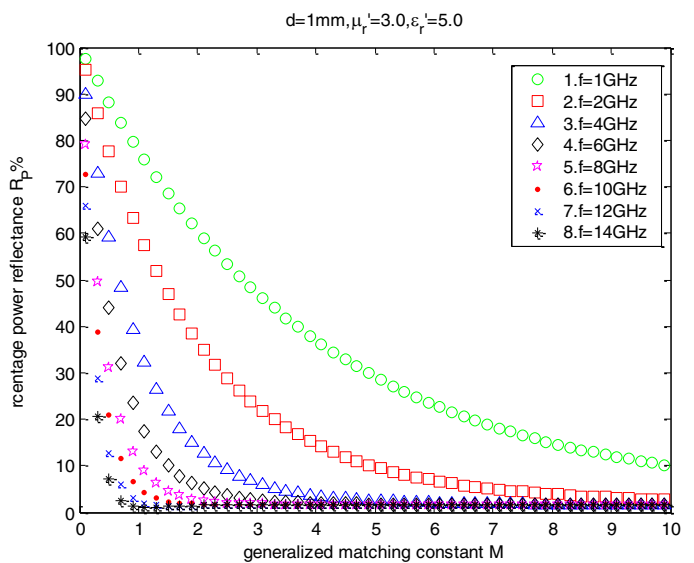

Fig.5. The RP $\%-M$ curves

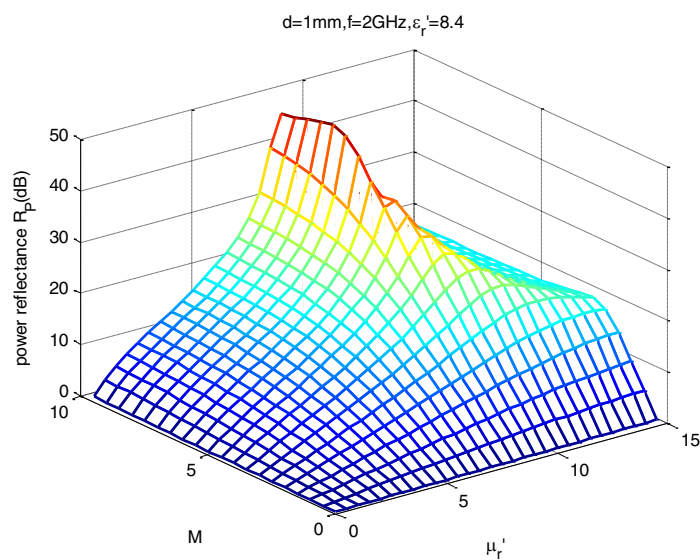

Fig.6. The $\mathrm{R}_{\mathrm{P}}(\mathrm{dB})-\mu_{\mathrm{r}}^{\prime}-\mathrm{M}$ grid curves

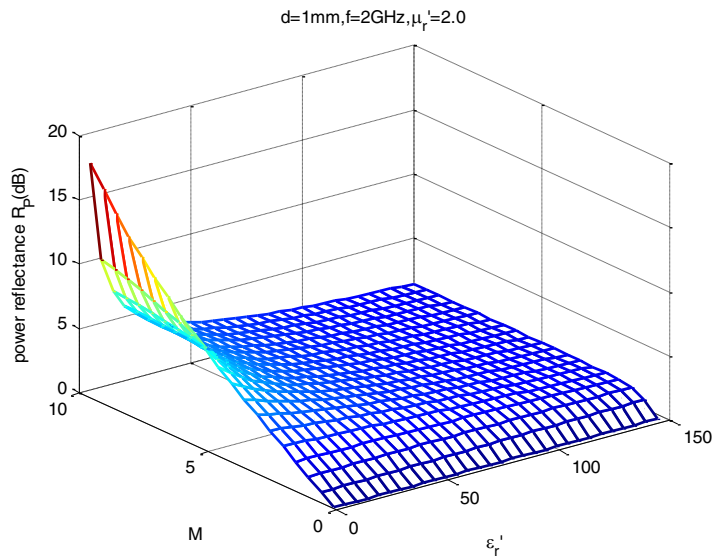

Fig.7. The $\mathrm{RP}(\mathrm{dB})-\varepsilon^{\prime}-\mathrm{M}$ grid curves

In figure 6 , with the increasing of $\mu_{\mathrm{r}}^{\prime}$, the $\mathrm{R}_{\mathrm{P}}(\mathrm{dB})$ peak will be emerged. And at $\mu_{\mathrm{r}}^{\prime}$ taking certain a value within the peak, the increasing $M$ makes the $R_{P}(d B)$ increase significantly, and finally becomes constant. While, at small $\mu_{\mathrm{r}}^{\prime}$ and $\mathrm{M}$ value, there are relatively downward-sloping and flat grid lines. In Figure 7, just at small $\varepsilon_{r}^{\prime}$ and larger $M$ value, the value of $\mathrm{R}_{\mathrm{P}}(\mathrm{dB})$ will be 
greater. All of them prove the view proposed in the PAM again.

Using the data in the reference 5 , and taking $\mu_{\mathrm{r}}^{\prime}=3$, $\varepsilon_{\mathrm{r}}^{\prime}=2, \mathrm{M}=5, \mathrm{~d}=1 \mathrm{~mm}$, and $\mathrm{f}=1 \mathrm{GHz}$, we calculate $\mathrm{R}_{\mathrm{P}}=5.51 \mathrm{~dB}$. However taking $\mathrm{f}=2 \mathrm{GHz}$ and the value of $\mu_{\mathrm{r}}^{\prime}$, $\varepsilon_{\mathrm{r}}^{\prime}, \mathrm{M}$ and $\mathrm{d}$ as the above, we calculate $\mathrm{R}_{\mathrm{P}}=11.38 \mathrm{~dB}$. This reflectivity can completely meet the currently military requirements on the RAM.

There are a few design parameters to be discussed, so the theoretical formula is only given in the multi-layer situation, and, by means of the computer, the specific material design can be carried out.

\section{Acknowledgment}

Authors acknowledge the funding support from national natural science foundation project of People's Republic of China(No.51141009), science and technology research project of Jiamusi university of People's Republic of China (No.Lz2013-017) and the basic scientific research operating expenses of Heilongjiang provincial universities of People's Republic of China(No. 2018-KYYWF-0956) for current research.

\section{References}

1. Zhuangjun Fan, Guohua Luo, Zengfu Zhan, Mater. Sci. Eng. B 132, 85(2006).

2. Kim S S,Jo S B,Gueon K I,et al. IEEE Trans. Magn. 27,5462(1991).
3. Miyata Y.IEEE Trans. Magn. 33,3427(1997).

4. T. J. Cui, C. H. Liang, IEEE Trans. Antennas Propagat. 42,621(1994).

5. Magali S P,Maria L G,Regina C R N,et al. Polym. Degrad. Stab.73,1(2001).

6. Zhi Gang Fang et al. 2010, Appl. Mech. Mater. 26, 246(2010).

7. Wang Y,Afsat M N .Microw. Opt. Techn. Let. 42,458(2004).

8. R. Mittra, D. Bouche, J. Pesque. IEEE Trans. Microwave Theory Tech. ,40,1789(1992).

9. Hossein Mosallaei,Yahya Rahmat-Samii. IEEE Trans. Antenn. propag. 48,1594(2000).

10. H. Cory, S. Shiran, M. Heilper.IEEE Trans., Electromag., Compat.35,451(1993).

11. J.A.Kong, J. Appl. Phys. 55,1364(1984).

12. P. Marquardt, L. Brngen, et al. Phys. Lett. A, 39,114(1986).

13. Viau, Raval, Fievet-Vincent. J. Magn. Magn. Mater. 140,401(1992).

14. L. Chateaau, G. Deleuze, Forveille. J. Appl. Phys. 73,3035(1993).

15. Shimizhu Y, et al. IEEE Trans. EC.E62,274(1979)

16. Richmond J H. IEEE Trans. Antenn. Propa. AP,35(1987).

17. 17Meyer F J C. Davidson D B. Microw. Opt. Techn. Let.7,1148(1994). 